\title{
The coronavirus pandemic should strengthen governments' will to invest in adolescent health
}

\author{
Agis Tsouros ${ }^{1,2}$
}

Received: 25 September 2020 / Accepted: 28 September 2020 / Published online: 14 October 2020

(C) Swiss School of Public Health (SSPH+) 2020

The pandemic offers a unique opportunity to all countries to change the way they think about and deal with health. Health is not high on the political agenda of countries, and public health systems are generally underfunded and weak as governments give priority to healthcare services. The accumulation of knowledge and evidence on the determinants of health and health inequalities has provided the solid ground for a whole of government and whole of society approach to health and well-being. But these remain elusive and hard to reach goals for the majority of countries. Health continues to be dominated by policy and action frameworks which cannot effectively accommodate the implementation of twenty-first century approaches to health development.

When it comes to addressing the health needs of population groups, child and adolescent health should be a priority for all countries. Most of the determinants of health, the social, economic, environmental, commercial, urban and cultural, are relevant to creating the conditions and the supportive environments for healthy children. Issues such as mental health including bullying, injuries and violence, nutrition, physical activity and obesity, addictions and social isolation to be effectively tackled require multi-sectoral actions and the active involvement of the community. Traditional approaches employed by the health and educator sectors alone are not sufficient.

Health is ultimately a political choice. It calls on leaders to determine the kind of society they wish to create. Investing in the health of children and adolescents is

This Editorial is part of the special issue Adolescent health in Central and Eastern Europe.

\footnotetext{
Agis Tsouros

tsouros@gmail.com

Imperial College London, London, UK

2 Present Address: 30 Rue des Salenques, 31000 Toulouse, France
}

investing in the future. The coronavirus pandemic showed the significance of political will and the need to engage all sectors and levels of government. Public health action is always underpinned by politics, consensus building (diplomacy) and scientific facts. Key public health concepts such as health in all policies, health literacy, population-based and life-course approaches are at the heart of action to promote adolescent health. Countries would need to create the pre-conditions to make these happen. These include putting health high on the social and political agenda; committing to reducing inequalities and addressing childhood poverty; strengthening governance for health at national and local levels; and empowering children, families and communities.

The UN sustainable development goals agenda offers the political legitimacy and the global impetus to seriously invest in the health and well-being of young people and future generations. Thinking about adolescents' health today should also include giving children skills for life, coping with uncertainty, becoming vocal about issues that affect life on the planet, addressing child abuse and discrimination, embracing cultural diversity, ensuring universal coverage to high-quality health services, social support and education and above all addressing childhood poverty and deprivation.

Identifying adolescent's health as a priority in countries is common. The same applies to all the factors and the conditions that determine their health. The key issue is not just to identify the correct priorities but most importantly to employ the best available evidence-based solutions and concepts and strive for maximum impact. For instance, addressing childhood obesity and limiting action to nutritional advice, health education and more physical activity in schools will not tackle this huge problem that has reached epidemic levels worldwide. Policies and interventions do not address the commercial determinants of health as well as the social and environmental conditions that are not conducive to healthy living for all children. Healthy urban planning and building healthy 
neighborhoods are critical in creating conditions for safe walking and cycling, access to greens and to open spaces for play and social interaction. Health literacy for children, parents, professionals and communities opens up tremendous opportunities for creating healthier societies and institutions. The well-known motto 'making the healthy choices - the easy choices' from the Ottawa Charter on Health Promotion is especially relevant to children in every respect including access and affordability.

The coronavirus pandemic is a wake-up call for the international community. It has shown how fragile economies and societies are and how existing inequalities are further exacerbated by such crises. The understanding of vulnerability and social disadvantage in communities was crucial in the management of the pandemic especially at the local level. The day after of this global crisis requires a wholehearted commitment to investing in and strengthening all aspects of public health: leadership, governance, operations including health promotion, disease prevention and protection, surveillance and preparedness and response to deal with emergencies. Preparedness to deal with public health emergencies will become increasingly relevant especially in relation to climate change. Giving a healthy start in life to children and creating the conditions and the services for healthy adolescence require strong and visionary leadership and a true understanding of the significance of health, equity and well-being in shaping the future of human societies.

Publisher's Note Springer Nature remains neutral with regard to jurisdictional claims in published maps and institutional affiliations. 